\title{
Scotopic Process and On-Elements in Human Retina.
}

\author{
By \\ Koiti Motokawa and Mituru Ebe. \\ （本川，弘 $\rightarrow ）$ (江. 部 充） \\ (From the Physiological Laboratory of Prof. K. Motokawa, \\ Tohoku University, Sendai.) \\ (Received for publication, January 11, 1951)
}

\section{INTRODUCTION.}

It is generally believed that the scotopic process is slower than the photopic process. This view is based on the facts that the time-course of dark adaptation is slower than that of light adaptation, and that the fusion frequency of flicker is lower at the periphery than at the fovea. ${ }^{11}$ From his studies on the time-course of electrical excitability of the eye after exposure to various colored lights, Motokawa ${ }^{2)}$ concluded that the red process is most rapid, the yellow and green processes intermediate, and the blue process slowest. The so-called rod-process which was analyzed by the same method $^{3)}$ was found to be more rapid than the blue process against the suppasition that the rod-process should be slower than any cone-process. The present investigation was undertaken to study more fundamentally the question as to whether or not the rod-process is really the slowest process in the human retina.

\section{EXPERIMENTAL.}

\section{Method and Results.}

In the present experiment the same method was used as was described in the paper cited above2). After a preliminary dark adaptation of about 20 minutes the electrical excitability of the eye was measured at varying moments after the end of pre-illumination. The electrical excitability was expressed in terms of a physiological quantity $\zeta$ which is defined as $\zeta=100\left(E-E_{0}\right) / E_{0}$, where $E$ and $E_{0}$ are reciprocals of electric thresholds determined with and without pre-illumination.

A circular patch of $2^{\circ}$ in visual angle was used for pre-illumination. For further details the paper cited above should be consulted.

In the majority of previous experiments we fixed the duration of light stimuli at 2 seconds, for it was known from preliminary experiments by Motokawa that the duration of pre-illumination has no effect upon the 
time-course of the electrical excitability of the eye unless the intensity of light is too weak. It has, however, been found that in the periphery of the retina and under certain experimental conditions $\zeta$-time curves or excitability curves can depend upon the duration of pre-illumination.

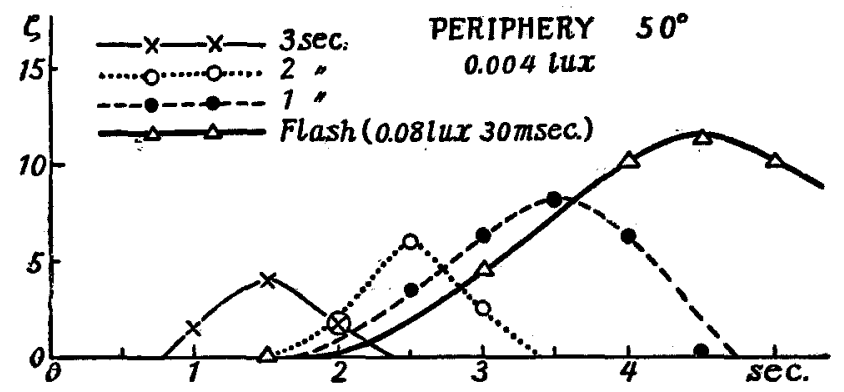

Fig. 1. Excitability curves for scotopic receptors. Ordinates: percentage increases in electrical excitability of the eye. Abscissae: time in seconds after the end of white pre-illuminating light. Duration of pre-illumination for each curve is given on top.

Such an example is shown in Fig. 1. In this experiment a retinal area $50^{\circ}$ from the fovea was exposed to white light of 0.004 lux for varying periods such as of $0.03,1,2$ and 3 seconds, and then changes in electrical excitability were measured in the manner stated above. Under these experimental conditions, i. e. at such a low intensity of pre-illumination and in such an extreme periphery of the retina, the photoreceptors involved must be scotopic receptors or rods; a small number of cones which might also be involved would play no important role. As can be seen in Fig. 1, the dependence on the duration of pre-illumination is such that the crest time of excitability curves as measured from the end of preillumination is lengthened as the duration of pre-illumination is decreased. Another aspect of dependence to be mentioned is that curves are higher with decreasing duration of pre-illumination. The crest times are about $1.5,2.5,3.5$ and 4.5 seconds for light stimuli of $3,2,1$ and 0.03 seconds respectively. It deserves attention that the sum of each crest time and the corresponding duration of pre-illumination remains invariant. This means that the time interval between the beginning of pre-illumination and the crest of the excitability curve remains constant irrespective of the duration of the light stimulus. This remarkable fact has an important bearing on the type of response of the scotopic elements. This relation will, however, more fully be discussed after further details of experimental results have been presented.

On the other hand, excitability curves for cones obtained at the fovea show no dependence upon the duration of pre-illumination, as has been confirmed again in the present experiment. Fig. 2 illustrates this relation; 


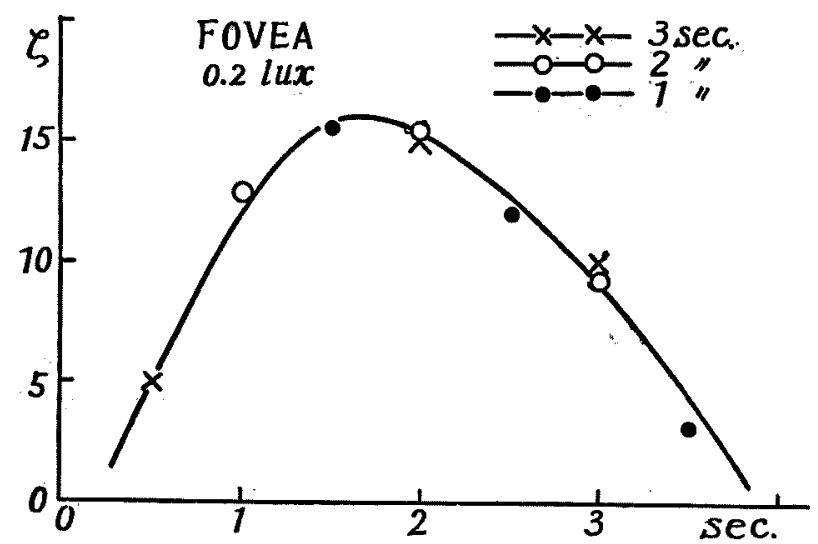

Fig. 2. Excitability curve for photopic receptors drawn with data obtained preilluminations of varying duration.

the data obtained with pre-illuminations of 1,2 , and 3 seconds can be represented by a single curve common to all kinds of data.

The phenomena observed above may be expressed in such a manner that the crest time of any excitability curve is independent of the duration of pre-illumination if the crest time is measured from the beginning of the light stimulus for the scotopic process, but from the end of it for the photopic process. In other words, the on-effects of the light stimulus must determine the time-course of the scotopic process, but the off-effects determine that of the photopic process.

Outside the fovea, where photopic and scotopic receptors co-exist, the crest time as measured from the end of pre-illumination either depends or does not depend upon the duration of pre-illumination according to whether the intensity of the pre-illuminating light is below or above the cone-threshold. When the intensity of light is sufficiently high, not only cones, but also rods must be excited, and one may suppose that the two kinds of receptors would equally contribute to an excitability curve determined under such conditions. As a matter of fact, however, the curve is determined by the photopic receptors alone, because $\zeta$-values of cones are generally much higher than those of rods. An example of an excitability curve obtained at the periphery $30^{\circ}$ from the fovea is illustrated in Fig. 3. The intensity of illumination was $10 \mathrm{lux}$, that is, much higher than the cone-threshold. The curve consists of two elevations, one at 1.5 sec, and the other at 3 sec. Based upon Motokawa's analysis ${ }^{2 / 3)}$, it can be said that the elevation at $1.5 \mathrm{sec}$. represents the yellow process (Y) while that at 3 sec. represents the blue process (B). This fact corresponds to the sensory fact that yellow and blue sensations are predominant in this region of the retina. It must be noted that this curve has been 


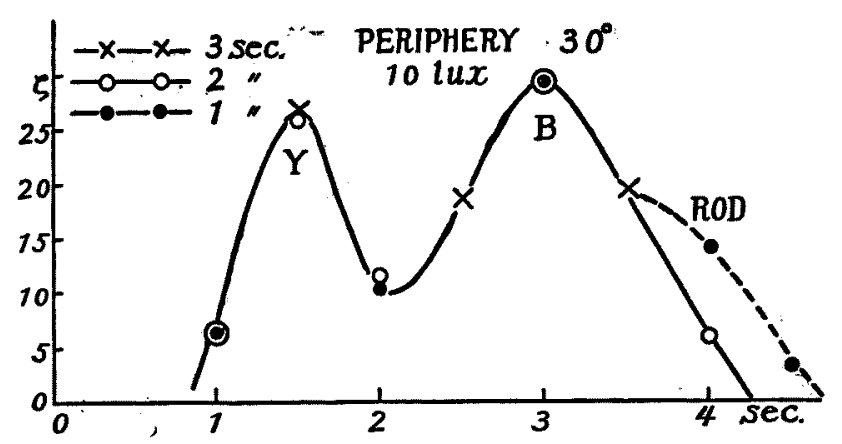

Fig. 3. Excitability curve obtained from periphery $30^{\circ}$ at a relatively high intensity of pre-illumination.

drawn with the data obtained with pre-illuminations of varying duration, and such a consistent result provides further evidence that the photopic process, wherever it may originate, depends little on the duration of pre; illumination. It must, however, be mentioned that towards the end of the curve some discrepancy is found between the $\zeta$-value obtained by illumination of 1 second and that obtained by illumination of 2 seconds; This difference is too great to be regarded as due to the error of measurements. The branch of the curve may, therefore, be supposed to be a manifestation of the scotopic process; as is obvious from Fig. 1, the scotopic process as determined by one second's pre-illumination has its maximum at $3.5 \mathrm{sec}$. and can survive every cone-process.

At a lower intensity of pre-illumination and in the periphery of the retina, the two kinds of process can manifest themselves in the same order of dominance, so that excitability curves obtained under such conditions can present very complicated patterns. Such cases are illustrated in Fig. 4.

The experiment was carried out at $50^{\circ}$ from the fovea and with light stimuli of $0: 2$ lux lasting for 1,2 and 3 seconds, The excitability curve for the duration of 3 seconds consists of two elevations, the one of which has a crest time of about $1.5 \mathrm{sec}$. and the other a crest time of about $3 \mathrm{sec}$ : (see continuous curve). Judged from the crest times, the former elevation must represent the process $\mathrm{Y}$, and the latter the process B. Thus, the photopic processes alone prevail when the duration of pre-illumination is relatively long, presumably because the scotopic curve is so low under these experimental conditions that it is covered by the process $Y$ (cf. Fig. 1). The earlier part of the curve for the duration of 2 seconds obviously. consists of the $Y$ process, but the later part presumably belongs to the scotopic process, because its maximum lies at about 2.25 seconds in close proximity to the maximum of.the scotopic process corresponding to the 


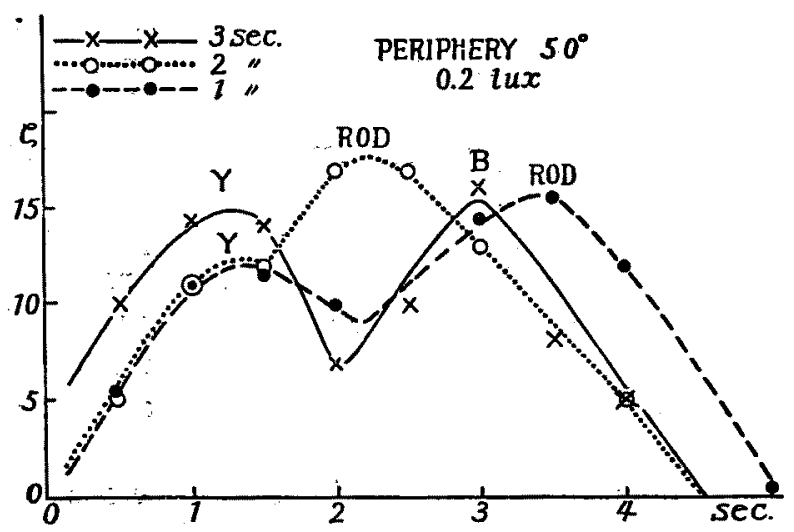

Fig. 4. Excitability curves obtained from periphery $50^{\circ}$ at an intensity of pre-illumination low but slightly higher than cone-threshold. Full explanation in text.

pre-illumination of 2 seconds in Fig. 1. The circumstance that there should be no photopic process having such a crest time in this region of the retina is another reason for identification of this maximum with the scotopic process. The curve for the duration of 1 second consists of two elevations, the one at-about $1.5 \mathrm{sec}$. and the other at $3.5 \mathrm{sec}$. The former is obviously the $\mathrm{Y}$ process and the latter the scotopic (cf. Fig. 1). From these examples it seems that it would be almost impossible for us to identify each elevation without the knowledge of the photopic and scotopic processes isolated in pure form.

With regard to the isolation of each retinal process the following remarks should be added. 1. For the isolation of the photopic processes it is advisable to use pre-illuminating light of sufficiently long duration so as to depress the appearance of the scotopic process. For this purpose, the duration of pre-illumination should be longer than 4 seconds. 2 . For the isolation of the scotopic process it is advantageous to use a flash of light for pre-illumination, because the scotopic excitability curve is highest and longest in duration under these experimental conditions so that it survives all photopic processes. It goes without saying that the isolation becomes more perfect if the intensity of light is reduced below the cone-threshold.

\section{Discussion.}

Hartline" found that discharges from a single optic nerve fiber fall into three main types, namely on-elements, off-elements and on/offelements, and this finding was confirmed by Granit with his micro-electrode technique. This author ${ }^{51}$ considers pure on-elements to be characteristic for rods, because of their dominance in the retina of the guinea pig which 
contains very few cones. Another important evidence for this view is that the distribution of spectral sensitivity of pure on-elements reproduces the pure visual purple curve (Donner and Granit ${ }^{6}$ ). According to Granit, on/off-elements together with pure off-elements seem to be in the majority in any eye containing cones.

Our data described above seem to be in complete agreement with these neurophysiological findings on animals. If rod-responses are of "on-type" as Granit considers, the onset of the pre-illuminating light should be the starting point of the scotopic excitability curve. If the scotopic process is characterized by its own time constant just as the photopic processes, it is expected that the crest time of the scotopic process as measured from the onset of pre-illumination should be constant irrespective of the duration of pre-illumination. Our experimental result is indeed just as expected from the neurophysiological finding by Granit.

Owing to our ignorance of the type of response of retinal elements we were quite at a loss when we found that the crest time of the so-called rod-curve as measured from the end of 2 seconds' illumination was shorter than that of the photopic process $B$, and that the crest time was found different with varying duration of the pre-illuminating light; because the former finding seemed difficult to be reconciled with the well established facts suggesting the slowness of the rod-process, and the latter appeared to contradict the law of characteristic time constants established on the cone-processes. Thanks to the significant suggestion by Granit, these questions have clearly been solved. It has been confirmed that the scotopic process is slower than any photopic process; the true crest time of the scotopic process is about 4.5 seconds while those for the red, yellow, green and blue processes are about 1, 1.5, 2 and 3 seconds respectively. Thus, our experiments have provided indirect evidence that Graint's statement applies to the human retina as well.

\section{Summary.}

Percentage increases in electrical excitability of the eye after exposure to white light are denoted by $\zeta$ :

1. At the fovea, $\zeta$-time curves or excitability curves depend little on the duration of pre-illumination; the time interval between the end of pre-illumination and the crest of the curve or crest time reamins constant irrespective of the intensity and duration of the light stimulus.

2. On the contrary, at the periphery of the retina and at a sufficiently low intensity of pre-illumination, the crest time generally depends on the duration of pre-illumination in such a manner that it is shorter with increasing duration. The crest time remains, however, independent of the duration of pre-illumination when it is measured from the beginning of 
the light stimulus.

3. This finding suggests that the change in electrical excitability in the scotopic mechanism is caused by the on-effects of illumination, and this interpretation agrees with Granit's animal experiments suggesting that on-elements are characteristic for rods.

4. The true crest time of the scotopic process is longer than that of any photopic process. In other word, the scotopic process is the slowest one in the retina.

\section{References.}

(1) Hecht, S. and Verrijp, C. D., J. Gen. Physiol., 1934, 17, 251.

(2) Motokawa, K., J. Neurophysiol., 1949, 12, 291.

(3) Motokawa, K., J. Neurophysiol., 1949, 12, 465.

(4) Hartline, H. K., Am. J. Physiot., 1938, 121, 400.

(5) Granit, R., Ergeb. d. Physiol., 1950, 46, 31.

(6) Donner, K. O. and Granit, R., Acta Physiol. Scand., 1949, 27, 161. 